\section{Femtosecond laser- assisted in situ keratomileusis for the correction of residual ametropia after deep anterior lamellar keratoplasty: a pilot investigation}

${ }^{1}$ From the Ophthalmology Unit, Department of Medicine, Surgery and Neuroscience, University of Siena, Siena, Italy

${ }^{2}$ Ophthalmic Hospital, Rome, Italy

${ }^{3}$ Studio Oculistico Associato Balestrazzi, Rome, Italy

Correspondence: A Balestrazzi, Dipartimento di Scienze Mediche, Chirurgiche e Neuroscienze, Università di Siena, Viale Bracci 1, via Berlinguer, Siena 53100, Italy Tel: +39 577585660; Fax: +39577586162. E-mail: angelo.balestrazzi@ unisi.it

Received: 15 January 2016 Accepted in revised form: 15 January 2017 Published online: 7 April 2017

\begin{abstract}
Purpose To evaluate the refractive and visual results of one-stage laser-assisted in situ keratomileusis with femtosecond laser (Femto-LASIK) for the correction of residual ametropia after deep anterior lamellar keratoplasty (DALK).

Methods This retrospective study included 13 eyes of 12 patients, who underwent corneal Femto-LASIK to correct refractive errors after DALK. IntraLase FS150 was used to create a lamellar flap and excimer laser ablation for refractive correction was performed using the Star S4 IR Excimer Laser in eight cases and the Technolas 217z Excimer Laser in Tissue Saving mode in five cases. Perioperative variables of interest included uncorrected (UDVA) and corrected (CDVA) distance visual acuities, manifest refraction and corneal topography.

Results The mean spherical equivalent preoperatively was $-4.85 \pm 2.82$ (range, -9.5 to $0 \mathrm{D})$. At the last visit the mean postoperative spherical equivalent (SE) was $-0.44 \pm 0.67 \mathrm{D}$ $(P<0.05)$. The mean postoperative UDVA was $0.12 \pm 0.12 \log$ MAR. There was a statistically significant increase in CDVA 6 months after Femto-LASIK $(P<0.05)$. Intraoperative and postoperative complications did not develop in this series of eyes.

Conclusions Correcting the preoperative manifest refractive error with Femto-LASIK led to a significant improvement in the UDVA and CDVA without surgical complications.

Eye (2017) 31, 1168-1175; doi:10.1038/eye.2017.44; published online 7 April 2017
\end{abstract}

\author{
A Balestrazzi ${ }^{1}$, A Balestrazzi ${ }^{2}, \mathrm{~F}$ Menicacci ${ }^{1}$, \\ G Cartocci ${ }^{1}$, F Menicacci ${ }^{1}$, P Michieletto ${ }^{2}$ \\ and E Balestrazzi ${ }^{3}$
}

\section{Introduction}

Deep Lamellar Anterior Keratoplasty (DALK) is a surgical technique which allows the removal of the external layers of the cornea preserving the recipient Descemet's membrane (DM) and endothelium. ${ }^{1}$ DALK offers the advantage of eliminating the possibility of allograft endothelial rejection, the most important complication of penetrating keratoplasty, and also makes it possible to reduce postoperative complications such as anterior synechia, secondary glaucoma or choroidal detachment. Because of these advantages, DALK has become the preferred method of surgical treatment for corneal diseases in which the endothelium is preserved, such as in keratoconus.

A variety of pre-descemetic and descemetic techniques for DALK have been introduced during the last recent years. ${ }^{2-7}$ Dissection of all stromal tissue overlying the recipient DM were found to create a smoother host bed ${ }^{8}$ and some studies suggest visual acuity or quality of vision differences between pre-descemetic and descemetic techniques. ${ }^{9-11}$

Similar to penetrating keratoplasty, postoperative refractive error is a frequent finding after DALK. One and two steps Microkeratome LASIK and more recently FemtoLASIK have been shown to safely and effectively reduce postoperative refractive error (in particular the spherical component) in patients who have previously undergone PK. ${ }^{12,13}$

To date, few reports have addressed photorefractive keratectomy (PRK) with or without use of mitomycin-C (MMC) $)^{14,15}$ and only one report has addressed laser in situ keratomileusis 
with microkeratome in eyes with previous DALK. ${ }^{16}$

Femtosecond laser-assisted in situ keratomileusis

(Femto-LASIK) used to correct refractive errors after

DALK has not previously been reported.

We report the refractive and visual results of 1-stage

Femto-LASIK after DALK.

\section{Materials and methods}

This retrospective non comparative clinical case series comprised 13 eyes of 12 patients, that could not be adequately corrected by contact lenses or spectacles, who underwent corneal Femto-LASIK between December 2010 and June 2014 to correct refractive errors after DALK.

All surgeries were performed by four experienced surgeons, at the Ophthalmology Unit, Department of Medicine, Surgery and Neuroscience, University of Siena, (AB and FM), Siena and at the Primavista Ophthalmic Center (EB and AlB), Rome, Italy.

The inclusion criteria were as follows: a clear deep anterior lamellar graft with an endothelial cell count (ECC) above 2000 cells/mm2, an optical pachymetry of $550 \mu \mathrm{m}$ or greater, a stable refractive error for at least 6 months after all sutures had been removed, and the absence of ocular pathology other than the indication for DALK. Exclusion criteria included significant haze at the host-donor interface, dry eye, collagen vascular disease, or diabetes. The patients were provided with comprehensive counseling preoperatively, including a full explanation of the risks associated with Femto-LASIK surgery. In particular, patients were informed of the investigational nature of Femto-LASIK in eyes with previous DALK.

\section{Preoperative evaluation}

Preoperatively, patients had a complete ophthalmologic examination including uncorrected distance visual acuity (UDVA), corrected distance visual acuity (CDVA), manifest refraction by auto-refraction (RM8800, Topcon, Tokyo, Japan), Javal-Schiotz keratometry, corneal topography and tomography, (Sirius, CSO, Florence, Italy) and Ocular Coherence Tomography (OCT) analysis and pachymetry by Visante OCT (Zeiss Meditec, Jena, Germany).

\section{Surgical technique}

IntraLase FS150 (Abbott Medical Optics, Inc., Irvine, CA, USA) was used to create a lamellar flap. The attempted thickness was $120 \mu \mathrm{m}$ in all cases. Femtosecond laser settings included a bed and side cut energy of $0.90 \mu \mathrm{J}$. In all cases the flap was planned with a diameter $0.2 \mathrm{~mm}$ smaller than the lamellar graft diameter and was centered inside the donor button, avoiding the graft-host junction.

After flap creation, the flap was lifted and excimer laser ablation for refractive correction was performed using the Star S4 IR Excimer Laser (Abbott Medical Optics, Inc.) in eight cases (Primavista, Rome) and the Technolas $217 \mathrm{z}$ Excimer Laser in Tissue Saving mode (Bausch and Lomb Surgical, Rochester, NY, USA) in five cases (Ophthalmic Units, Siena, Italy, Table 1). The postoperative treatment consisted of topical tobramycin $0.3 \%$ and dexamethasone $0.1 \%$ (To- braDex) three times daily for the first 7 days, two times for the second week, and one time for 1 month. Preservative-free artificial tears (sodium hyaluronate) were used up to 6 months in each case.

\section{Postoperative evaluation}

All eyes were examined postoperatively at 1 day, 1 week, and 1 and 6 months. The examinations included UDVA, CDVA, manifest refraction and intraocular pressure. Outcome measures were recorded at the last postoperative visit and included UDVA, CDVA, manifest refraction, corneal topography and OCT analysis and pachymetry, performed using the same devices that were used preoperatively.

\section{Statistical analysis}

All data were collected in an Excel database (Microsoft Office for Mac 2011; Microsoft, Redmond, WA, USA) and analyzed with SPSS for Mac (version 16.0, SPSS, Inc., Chicago, IL, USA). Snellen UDVA and CDVA were converted into LogMAR for the mathematical and statistical calculations. The mean and standard error of the mean were calculated for the following variables: age, preoperative and postoperative CDVA, preoperative spherical equivalent (SE), postoperative SE, and postoperative UDVA. Paired samples $t$-tests were used to analyze preoperative and postoperative visual acuity, astigmatism and SE parameters. Differences were considered statistically significant when the $P$-value was less than 0.05 .

\section{Results}

The study enrolled 13 eyes of 12 patients. The mean age of the 5 women and 7 men was 36.5 years \pm 8.14 (SD) (range 29-52 years). Table 2 shows the patients' demographics.

The mean time between DALK and LASIK was $28.3 \pm 9.7$ months (range 16-44 months). There were no intraoperative or postoperative complications. Patients were followed for a mean of $21.1 \pm 6.6$ months (range 12-38 months) after Femto-LASIK.

Table 1 shows the visual and refractive outcomes. The mean spherical equivalent preoperatively was 
Table 1 Visual and refractive outcomes

\begin{tabular}{lccr}
\hline & Preop. & Prostop. & P-value \\
\hline UDVA (LogMar) & & & \\
Mean \pm SD & $1.04 \pm 0.35$ & $0.12 \pm 0.13$ & $<0.001$ \\
Range & $0.4-1.8$ & $0-0.3$ & \\
& & & \\
Cylinder (D) & & & \\
Mean \pm SD & $-4.35 \pm 1.90$ & $-0.92 \pm 1.39$ & $<0.001$ \\
Range & $1.25-7$ & $0-3$ & \\
SE (D) & & & \\
Mean \pm SD & $-4.58 \pm 2.82$ & $-0.44 \pm 0.67$ & $<0.001$ \\
Range & $1-9.5$ & $0-1.5$ & \\
& & & 0.002 \\
CDVA (LogMar) & & & \\
Mean \pm SD & $0.12 \pm 0.12$ & $0.02 \pm 0.03$ & \\
Range & $0-0.4$ & $0-0.1$ & \\
\hline
\end{tabular}

Table 2 Patients' demographics

\begin{tabular}{lr}
\hline Eyes $(n)$ & 13 \\
Age $(y)$ & 36.5 \\
Mean & $27-52$ \\
Range & \\
& \\
Sex $(\mathrm{n})$ & 7 \\
Male & 5 \\
Female & \\
Transplantation indication & 11 \\
Keratoconus & 1 \\
Corneal scar & \\
Refractive surgery indication & 7 \\
Anisometropia \\
Contact lenses intolerance \\
$\quad$ Glare
\end{tabular}

$-4.85 \pm 2.82$ (range, -9.5 to $0 \mathrm{D}$ ). The mean preoperative astigmatism was $-4.25 \pm 1.9(-7.00$ to $-1.25 \mathrm{D})$. In the 13 eyes the preoperative uncorrected visual acuity (UCVA) was $1.07 \pm 0.35$. Preoperative CDVA ranged between 0.2 and 0 logMar.

The mean postoperative UDVA was $0.12 \pm 0.12$ $\log$ MAR (Figure 1). There was a statistically significant increase in CDVA 6 months after Femto-LASIK $(P<0.0005)$. The postoperative CDVA was $0.00 \log$ MAR in 9 eyes $(69.2 \%)$ and $0.05 \log$ MAR in 2 eyes $(15.4 \%)$ and 0.1 in 2 eyes (15.4\%).

The mean preoperative manifest sphere and cylinder was $-2.34 \pm 3.37$ and $-4.35 \pm 1.90 \mathrm{D}$, respectively. The mean postoperative manifest sphere and cylinder refraction was $-0.00 \pm 0.39$ and $-0.92 \pm 1.38 \mathrm{D}$, respectively. The attempted astigmatic correction was not totally achieved in all cases (Table 3). There was a

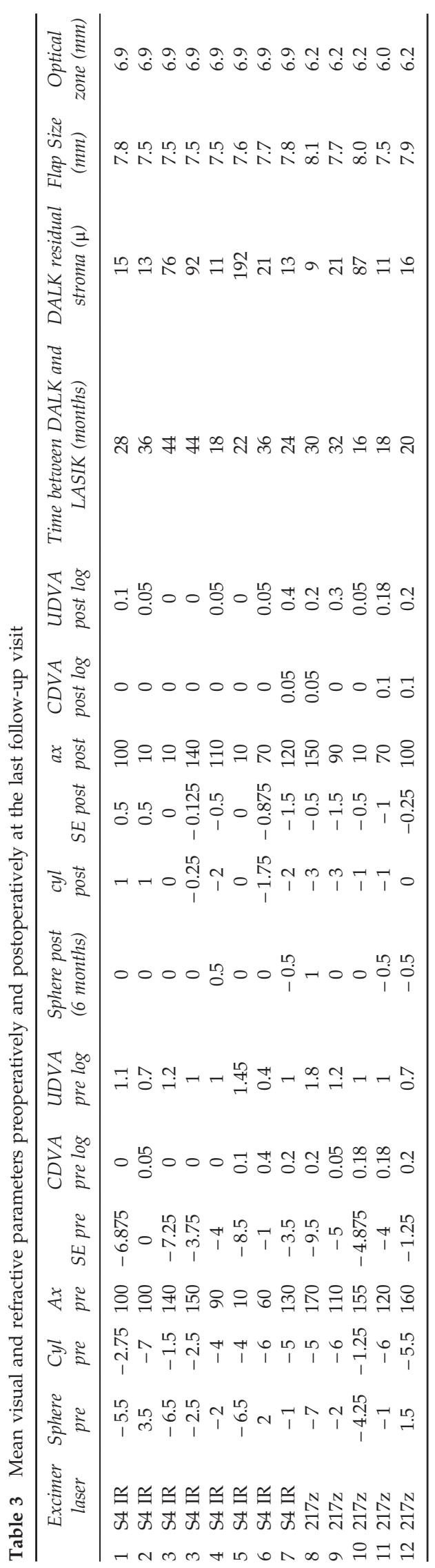


significant reduction in refractive sphere and astigmatism 6 months after Femto-LASIK $(P<0.002)$.

The mean preoperative spherical equivalent (SE) in all 13 eyes was $-4.57 \pm 2.82 \mathrm{D}$. At the last visit the mean postoperative SE was $-0.44 \pm 0.67 \mathrm{D}$. The difference between preoperative and postoperative SE was statistically significant $(P<0.002)$. SE was within $0.25 \mathrm{D}$ of plano in four eyes $(30.77 \%)$, from 0.25 to $0.50 \mathrm{D}$ of plano in five eyes $(38.45 \%)$, and within $1.5 \mathrm{D}$ of emmetropia in four eyes (30.77\%).

Figure 2 shows the standard graphs for reporting outcomes of refractive surgery.

Intraoperative and postoperative complications such as wound dehiscence, buttonhole, partial flap, and graft rejection did not develop in this series of eyes.

The topographical preoperative and postoperative patterns of 2 cases are reported on Figure 1.

Table 3 shows the mean visual and refractive parameters preoperatively and postoperatively at the last follow-up visit.

\section{Discussion}

DALK has gained popularity as a treatment option for eyes with healthy endothelium because it has several advantages over PK. ${ }^{1}$ One of the main problems with DALK surgery has been reported to be the inability to obtain a smooth interface between donor tissue and recipient DM. ${ }^{10,17}$ This led to stromal scarring and interface opacities, especially when layers of host stroma remained on the recipient DM. Abdelkader and Kaufman, analyzed the interface healing process and keratocyte status after 2 types of DALK with and without exposure of DM by confocal microscopy. ${ }^{8}$ These events were correlated with the clinical haze observed postoperatively and demonstrated in the Descemetic group, that the apposition of the smooth donor stromal surface to the smooth host DM resulted in less bright and less reflective activated keratocytes and hence less haze. The interface quality may be responsible for differences in visual acuity between pre-descemetic and descemetic techniques. In fact, despite some authors, such as Sarnicola ${ }^{9}$ reporting no differences of visual acuity after a 30 months follow up between pre-descemetic and big bubble descemetic eyes, other studies, such as Fontana ${ }^{10}$ state that eyes with successful big bubble show greater CDVA than those that required manual dissection at follow-up $\leq 5$ years.

LASIK has been widely used since its inclusion in the clinical routine in the early 1990s to treat a wide range of

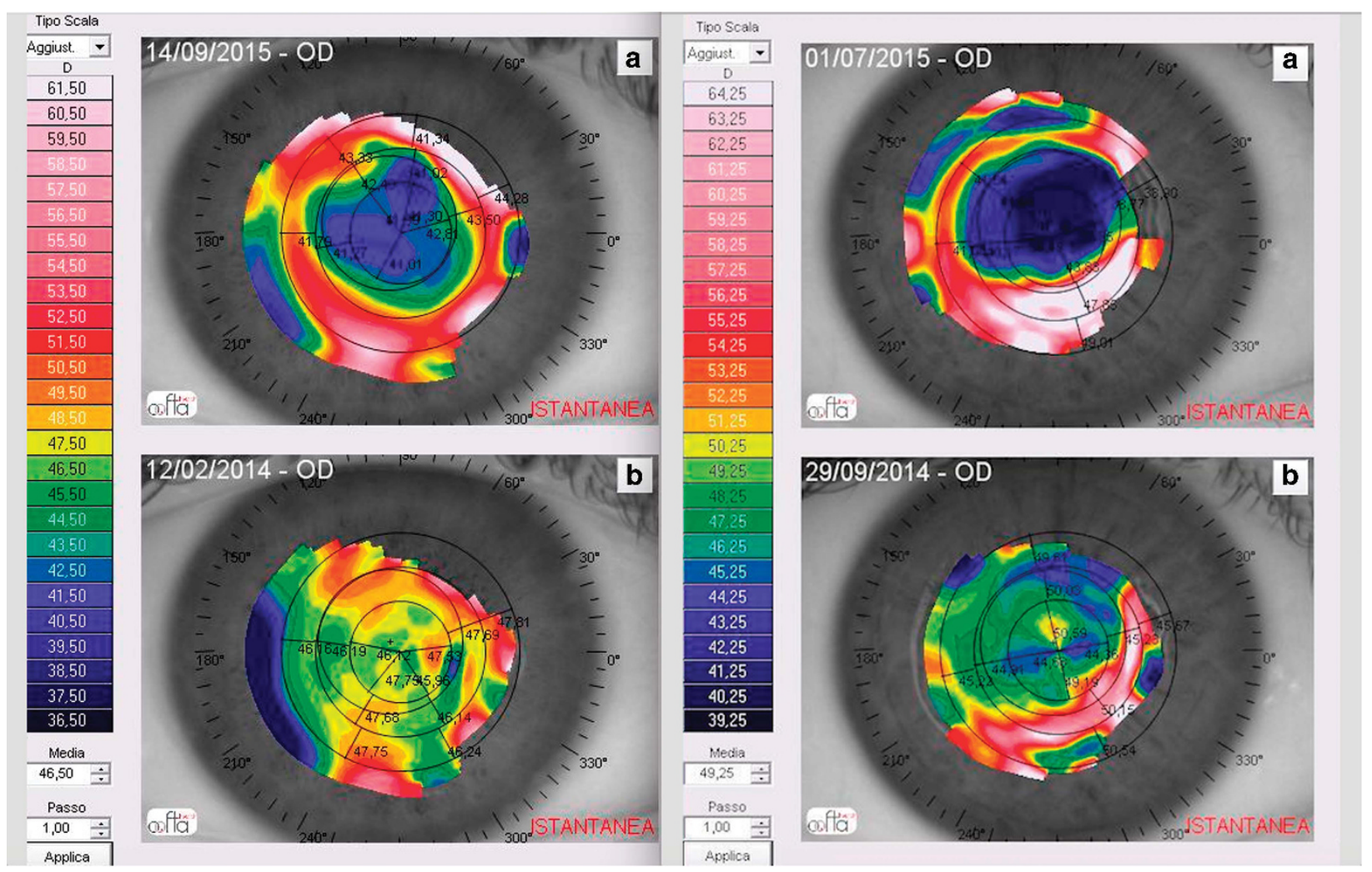

Figure 1 Postoperative (a) and preoperative (b) corneal topography of two cases. The centration is good and the optical zone is sufficient. 
refractive disorders. LASIK combines a high patient convenience due to the lack of postoperative complaints and fast visual recovery with predictable, precise, and stable results. ${ }^{18}$ Both microkeratome ${ }^{19,20}$ and FemtoLASIK have been shown to be effective in the correction of post-PK ametropia, but Femto-LASIK offers several

a

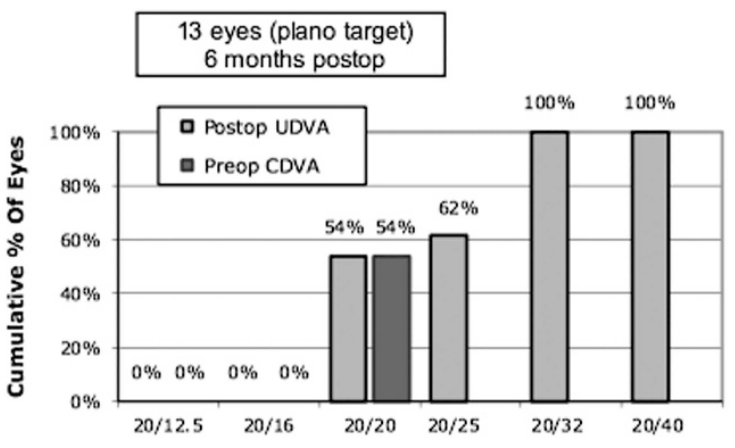

Cumulative Snellen Visual Acuity ( $20 / x$ or better)

\section{Uncorrected Distance Visual Acuity}

c

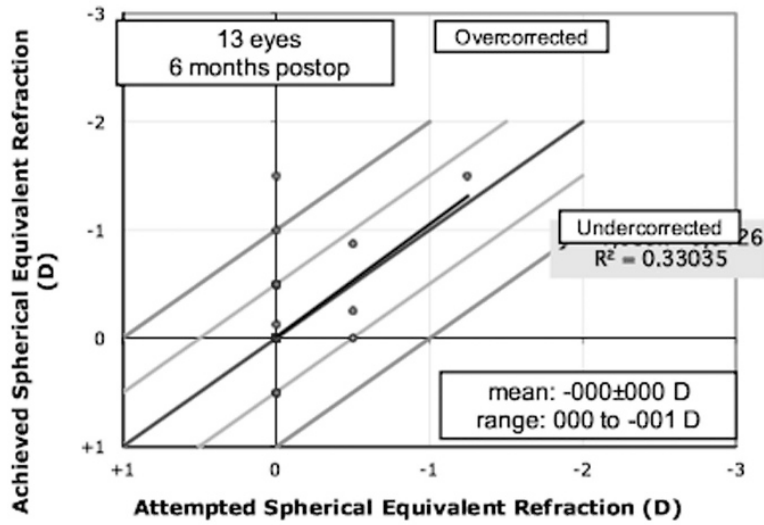

Spherical Equivalent Attempted vs Achieved

e

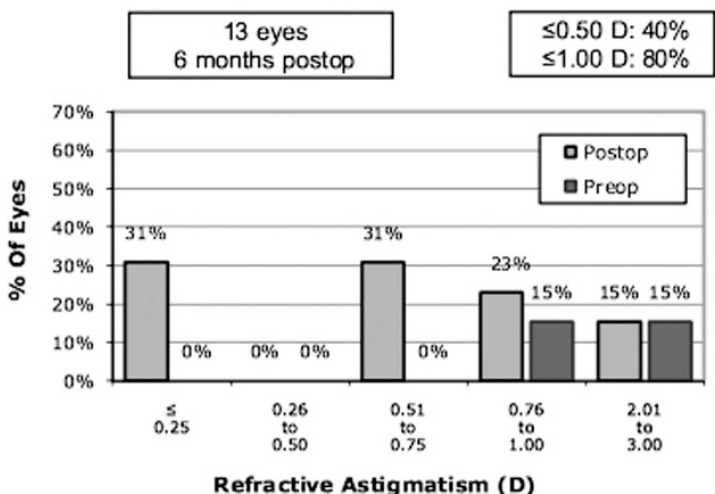

Refractive Astigmatism benefits that include more predictable flap thicknesses and planarity, the possibility to choose the flap centration, and overall fewer intraoperative flap complications, especially in case of high corneal dioptric power. ${ }^{21}$

Only a few papers investigate the results of this technique after Deep Anterior Lamellar Keratoplasty.

b

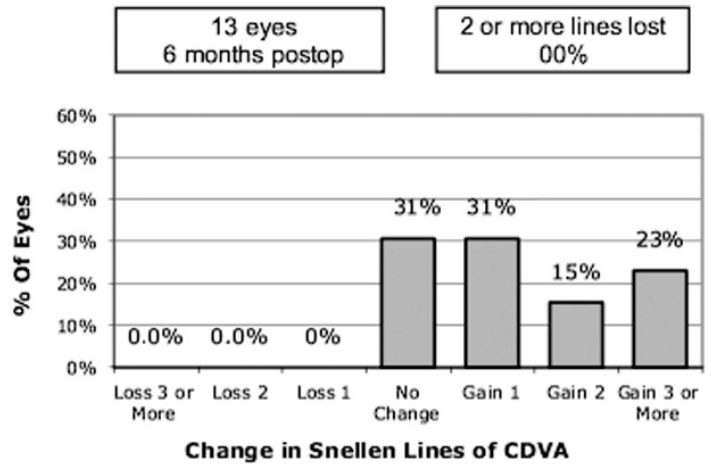

Change in Corrected Distance Visual Acuity

d

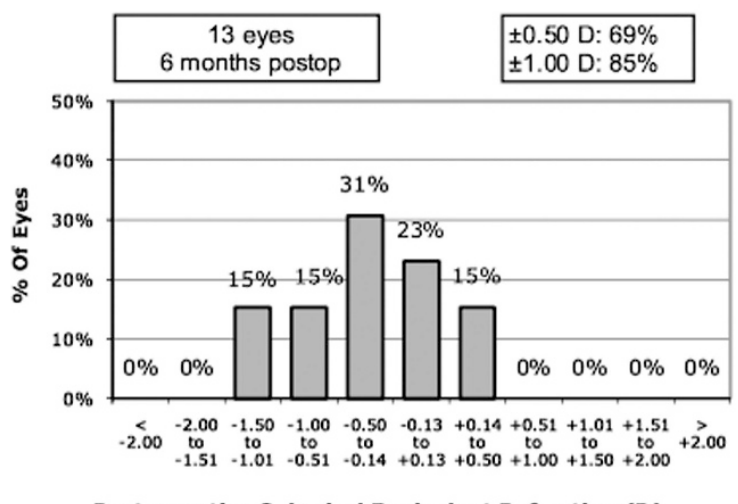

Spherical Equivalent Refractive Accuracy

f

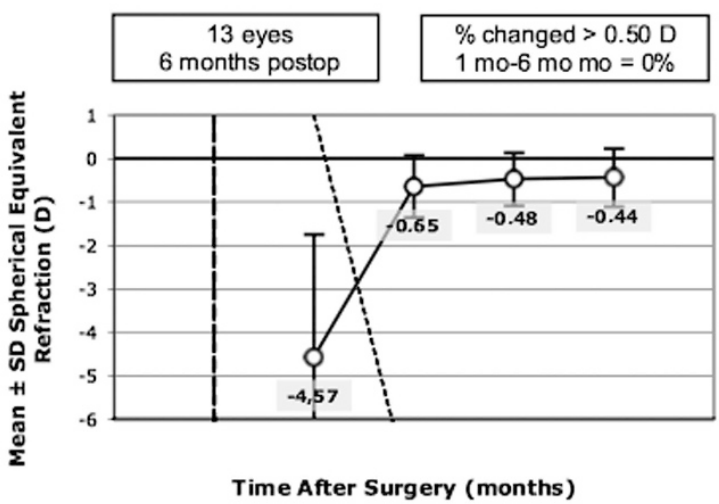

Stability of Spherical Equivalent Refraction

Figure 2 (a) Preoperative and postoperative UDVA. (b) Changes in CDVA with Femto-LASIK after DALK. (c) Refractive predictability of Femto-LASIK after DALK. (d) Accuracy of SE refraction. (e) Preoperative and postoperative refractive astigmatism. (f) Stability of SE refraction. 
Banu Toru Acar recently reported good refractive results after either 1-step or 2-step microkeratome LASIK procedures in a retrospective 13 eyes case series. ${ }^{22}$ To our knowledge, this is the first paper on the application of Femto-LASIK for ametropia correction in post-DALK patients using the tissue- saving or customvue or standard visx treatment. This series has demonstrated that Femto-LASIK after a corneal lamellar graft can be a safe and effective procedure. Our results show excellent safety. No complications were observed during corneal flap creation and laser ablation. No wound healing problems or graft rejection occurred during the follow-up.

Regarding the visual results, a significant improvement in UDVA, CDVA, cylinder and SE reduction was found. Most eyes maintained their CDVA and some gained multiple lines of CDVA (Figure 3). This is an important finding, especially considering that several studies suggest postoperative visual limitations after predescemetic DALK due to the interface quality and to the recipient stromal thickness. ${ }^{8}$ In our case series, also in predescemetic DALK, an important visual acuity improvement occurred, and in one case, with a recipient bed thickness greater than 100 microns, a 0 LogMar postoperative UDVA was obtained, with a two LogMar lines improvement in spite of a double interface (Figure 4).

Banu Toru Acar recently also reported ${ }^{22}$ that irregular astigmatism, which is almost always present after penetrating or lamellar keratoplasty, could translate into very high levels of high-order aberrations (HOA) that are difficult to quantify with total wavefront analysis. This finding could affect the visual results after Femto-LASIK. On the other hand, authors, such as McLaren ${ }^{23}$ did not

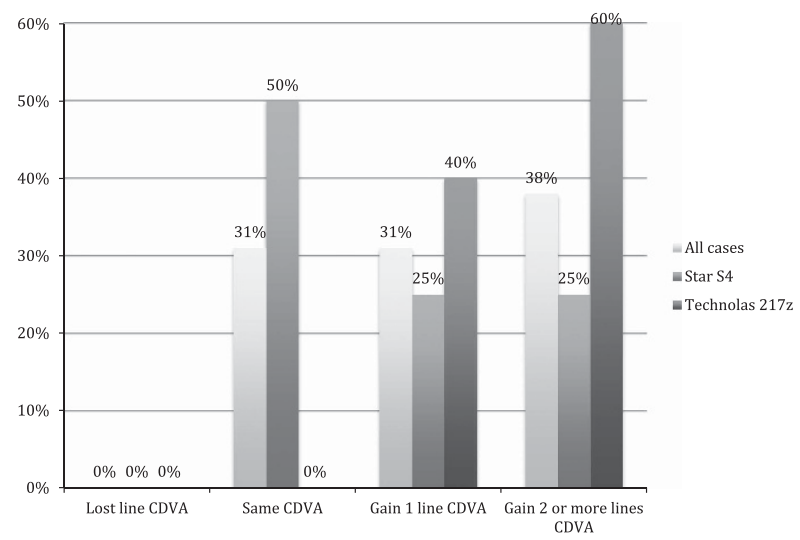

Figure 3 Change in Corrected Distance Visual Acuity 6 months after Femto-LASIK.
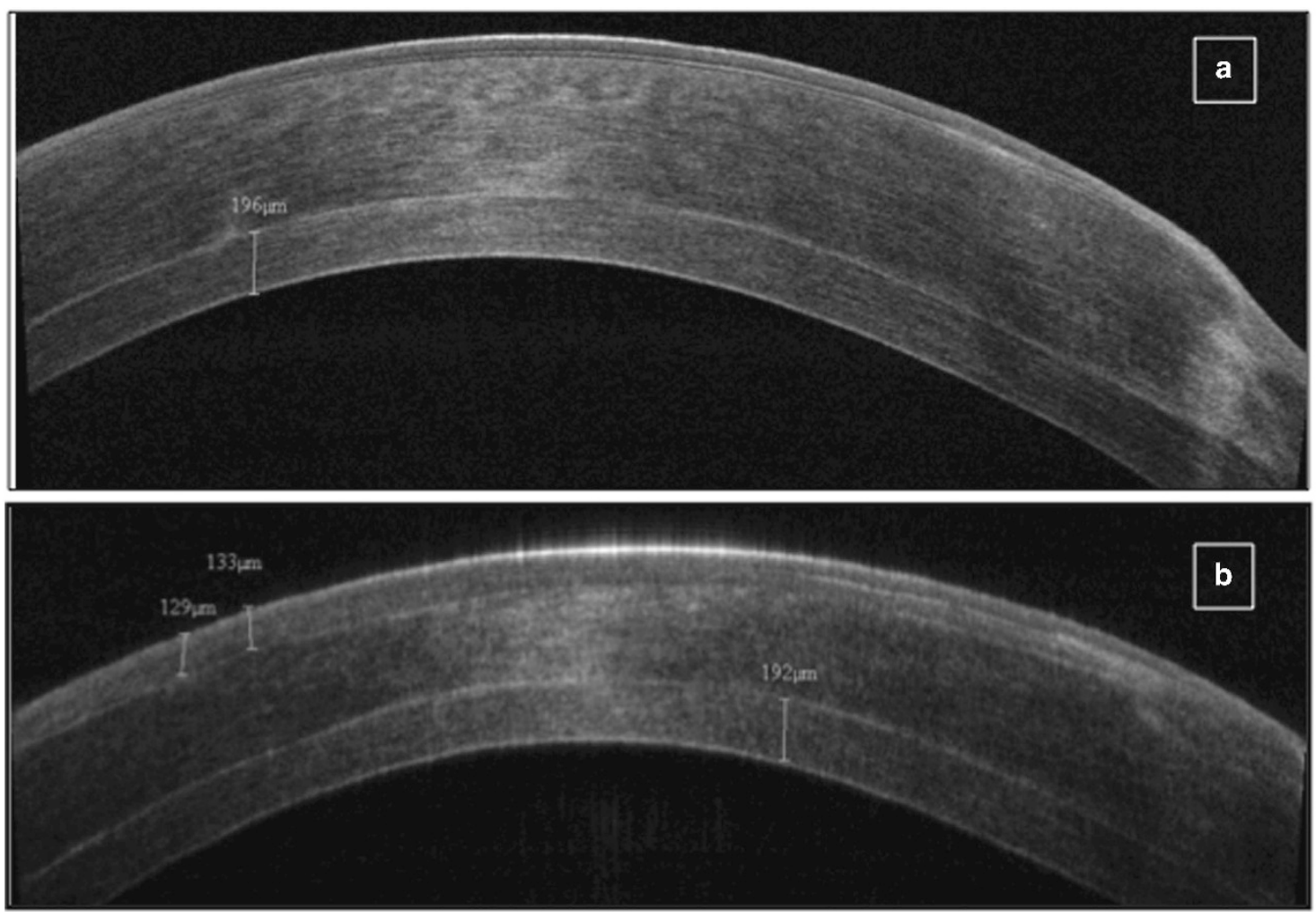

Figure 4 Preoperative (a) and postoperative (b) case 8 corneal OCT. In spite of a double interface and of a 192 microns recipient bed thickness, final UDVA and CDVA was 0 logMar. 
find a correlation between corneal higher-order wavefront errors and visual function in post- keratoplasty eyes. In our opinion the main goal of Femto-LASIK after keratoplasty is first of all to obtain a good visual acuity and also the anisometropia resolution. The HOA improvement could be a secondary and misleading result, especially considering that the patient treated are affected by corneal diseases that invariably cause abnormal HOA.

This study has limitations that must be taken into consideration when interpreting the results. Importantly, the sample size was small and it represents the results at two centers with the same Femtosecond laser but with two different excimer lasers. Moreover the study is retrospective and patients were also not randomized for different treatments.

Furthermore it is important to underline that in the treated eyes suffering from keratoconus, there is a risk of ectasia re-emergence after 15-20 years. Thus additional intervention surgical and non-surgical may be needed for such eyes in the long run.

In conclusion, correcting the preoperative manifest refractive error with Femto-LASIK led to a significant improvement in the UDVA and CDVA without surgical complications. The treatment seems to be associated with acceptable residual refractive errors and represents a treatment option in post-DALK patients. Thus, prospective comparative studies with larger samples should continue to evaluate the efficacy of this procedure.

\section{Summary}

\section{What was known before}

- Both microkeratome and Femto-LASIK have been shown to be effective in the correction of post penetrating keratoplasty ametropia.

What this study adds

- This is the first paper on the application of Femto-LASIK for ametropia correction in post deep anterior lamellar keratoplasty patients using the tissue- saving or customvue or standard visx treatment. This series has demonstrated that Femto-LASIK after a corneal lamellar graft can be a safe and effective procedure.

\section{Conflict of interest}

The authors declare no conflict of interest.

\section{References}

1 Shimmura S, Tsubota K. Deep anterior lamellar keratoplasty. Curr Opin Ophthalmol 2006; 17: 349-355.

2 Tsubota K, Kaido M, Monden Y, Satake Y, Bissen-Miyajima H, Shimazaki J. A new surgical technique for deep lamellar keratoplasty with single running suture adjustment. Am J Ophthalmol 1998; 126: 1-8.

3 Sugita J, Kondo J. Deep lamellar keratoplasty with complete removal of pathological stroma for vision improvement. $\mathrm{Br} \mathrm{J}$ Ophthalmol 1997; 81: 184-188.

4 Melles GRJ, Lander F, Rietveld FJR, Remeijer L, Beekhuis WH, Binder PS. A new surgical technique for deep stromal, anterior lamellar keratoplasty. Br J Ophthalmol 1999; 83: 327-333.

5 Anwar M, Teichmann KD. Deep lamellar keratoplasty. Surgical techniques for anterior lamellar keratoplasty with and without baring of Descemet's membrane. Cornea 2002; 21: 374-383.

6 Caporossi A, Simi C, Licignano R, Balestrazzi A, Traversi C. Air-guided manual deep lamellar keratoplasty. Eur J Ophthalmol 2004; 14: 55-58.

7 Balestrazzi E, Balestrazzi AN, Mosca L, Balestrazzi A. Deep lamellar keratoplasty with trypan blue intrastromal staining. J Cataract Refract Surg 2002; 28: 929-931.

8 Abdelkader A, Kaufman HE. Descemetic versus predescemetic lamellar keratoplasty: Clinical and Confocal Study. Cornea 2011; 30: 1244-1252.

9 Sarnicola V, Toro P, Gentile D, Hannush SB. Descemetic DALK and predescemetic DALK: outcomes in 236 cases of keratoconus. Cornea 2010; 29: 53-59.

10 Fontana L, Parente G, Sincich A, Tassinari G. Influence of graft-host interface on the quality of vision after deep anterior lamellar keratoplasty in patients with keratoconus. Cornea 2011; 30: 497-502.

11 Baradaran-Rafii A, Eslani M, Sadoughi MM, Esfandiari H, Karimian F. Anwar versus melles deep anterior lamellar keratoplasty for keratoconus:a Prospective Randomized Clinical Trial. Ophthalmology 2013; 120: 252-259.

12 Aliò JL, Javaloy J, Osman AA, Galvis V, Tello A, Haroun HE. Laser in situ keratomileusis to correct post-keratoplasty astigmatism; 1 -step versus 2-step procedure. J Cataract Refract Surg 2004; 30: 2303-2310.

13 Buzard K, Febbraro J-L, Fundingsland BR. Laser in situ keratomileusis for the correction of residual ametropia after penetrating keratoplasty. J Cataract Refract Surg 2004; 30: 1006-1013.

14 Leccisotti A. Photorefractive keratectomy with mitomycin C after deep anterior lamellar keratoplasty for keratoconus. Cornea 2008; 27: 417-420.

15 Pedrotti E, Sbabo A, Marchini G. Customized transepithelial photorefractive keratectomy for iatrogenic ametropia after pene- trating or deep lamellar keratoplasty. J Cataract Refract Surg 2006; 32: 1288-1291.

16 Toru Acar B, Utine CA, Acar S, Ferda Ciftci F. Laser in situ keratomileusis to manage refractive errors after deep anterior lamellar keratoplasty. J Cataract Refract Surg 2012; 38: 1020-1027.

17 Mosca L, Fasciani R, Tamburelli C, Buzzonetti L, Guccione L. Mandara`E, and Balestrazzi E. Femtosecond Laser-assisted Lamellar Keratoplasty, Early Results. Cornea 2008; 27: 668-672.

18 Pallikaris IG, Siganos DS. Excimer laser in situ keratomileusis and photorefractive keratectomy for correction of high myopia. J Refract Corneal Surg 1994; 10: 498-510.

19 Arenas E, Maglione A. Laser in situ keratomileusis for astigma- tism and myopia after penetrating keratoplasty. J Refract Surg 1997; 13: 27-32. 
20 Spadea L, Mosca L, Balestrazzi E. Effectiveness of LASIK to correct refractive error after penetrating keratoplasty.

Ophthalmic Surg Lasers 2000; 31: 111-120.

21 Ghoreishi M, Naderi Beni A, Naderi Beni Z. Visual outcomes of Femto-LASIK for correction of residual refractive error after corneal graft. Graefes Arch Clin Exp Ophthalmol 2013; 251: 2601-2608.
22 Acan BT, Utine CA, Acar S, Ciftci F. Laser in situ keratomileusis to manage refractive errors after deep anterior lamellar keratoplasty. J Cataract Refract Surg 2012; 38: 1020-1027.

23 McLaren JW, Patel SV, Bourne WM, Baratz KH. Corneal wave-front errors 24 months after deep lamellar endothelial keratoplasty and penetrating keratoplasty. Am J Ophthalmol 2009; 147: 959-965. 